\title{
Effects of Alloy Composition on Multilevel Operation in Self-heating Phase Change Memories
}

\author{
S. Braga ${ }^{1}$, N. Pashkov ${ }^{2}$, L. Perniola ${ }^{2}$, A. Fantini ${ }^{2}$, A. Cabrini ${ }^{1}$, G. Torelli ${ }^{1}$, V. Sousa ${ }^{2}$, B. De Salvo ${ }^{2}$, and G. Reimbold ${ }^{2}$ \\ ${ }^{1}$ Department of Electronics, University of Pavia, 1, Via Ferrata, 27100, Pavia, ITALY, stefania.braga@unipv.it \\ ${ }^{2}$ CEA-LETI, MINATEC, 17, Rue des Martyrs, 38054, Grenoble, FRANCE, luca.perniola@,cea.fr
}

\begin{abstract}
Although PCM presents good scaling properties, multilevel storage may be required to increase bit density and reduce the cost per bit. The aim of this paper is to evaluate the impact of different phase change (PC) materials based on GST and GeTe on the feasibility of partial-RESET multilevel programming in self-heating device architectures. Through an accurate analysis of electrical and thermal characteristics of these devices, it is demonstrated that GeTeN10\% and GST appear to be the best candidates to assure multilevel operation in such architectures.
\end{abstract}

Keywords - Phase change memories, multilevel storage, phase change materials, self-heating, chalcogenide alloys

\section{INTRODUCTION}

In Phase Change Memories (PCMs), digital information is stored as the value of the electrical resistance of a thin layer of a chalcogenide alloy (typically, $\mathrm{Ge}_{2} \mathrm{Sb}_{2} \mathrm{Te}_{5}, \mathrm{GST}$ ) [1], which can be reversibly switched between the high-resistance state, so called RESET, and the low resistance state, so called SET state [2]. A difference of about two orders of magnitude exists between the electrical resistivities of the two states, which easily allows non-volatile bi-level storage and can in principle be exploited for multilevel (ML) operation. In the ML approach, a single memory cell can be programmed to any of $n=2^{b}(b>1)$ different resistance values and is then able to store $b$ bits, which leads to higher storage density and, hence, to lower cost-per-bit for any given fabrication technology generation. The nominal values of the intermediate programmed levels (i.e., the levels between the full-SET and

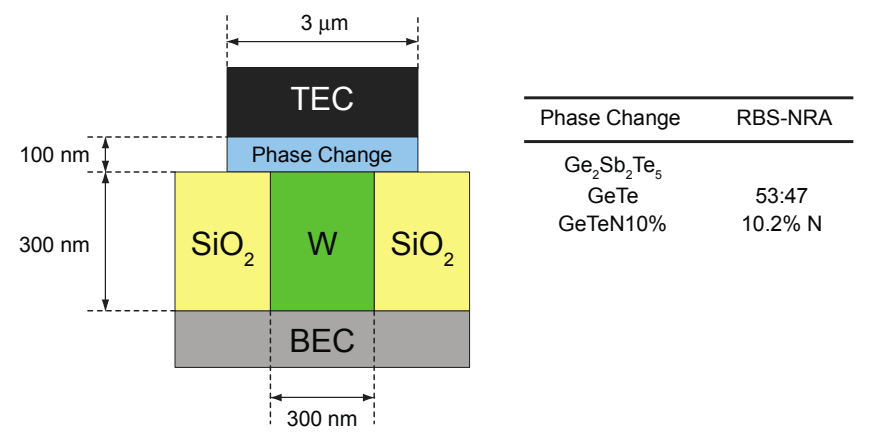

Figure 1. Schematic picture of the lance-type PCM cell architecture investigated in this work and details on the integrated phase change materials. Note that composition has been measured by Rutherford Backscattering Spectroscopy (RBS) and Nuclear Reaction Analysis (NRA) for nitrogen. the full-RESET state) are the result of a trade-off choice between several requirements, such as programming and read accuracy, reproducibility, and data retention. These properties strongly depend on the chalcogenide alloy composition. Although many efforts have been devoted to study the data retention properties of different alloys [3]-[5], effects of phase-change material characteristics on the feasibility of ML storage are still to be assessed.

In this paper, we study the feasibility of ML storage in PCM cells realized with different chalcogenide alloys. To this end, we focus on the staircase-up partial-RESET programming approach [6], which consists in applying partial-RESET pulses of increasing amplitude to the cell initially programmed to its minimum resistance state. In particular, we analyse the programming behaviour of the considered PCM cells and evaluate the key material parameters that affect the feasibility of partial-RESET ML storage.

\section{ELECTRICAL CHARACTERIZATION}

Our experimental investigation was carried out on PCM cells having a cylindrical tungsten heater with a diameter of $300 \mathrm{~nm}$, as shown in Fig. 1 (lance-type architecture). The phase change layer thickness is $100 \mathrm{~nm}$. In this work, we consider three different PC materials, namely: GST, GeTe, and N-doped GeTe (10\% doping), which will be referred to as GeTeN10\%.

The sequence of electrical pulses applied to the cells for characterization is shown in Fig. 2. First, the PCM cell is brought into the full-SET state by means of a staircase-down initializing procedure, then a sequence of cumulative staircaseup (SCU) pulses is applied in order to partially amorphize the PC layer. During each programming pulse, the current flowing through the cell was measured by means of our experimental setup. After each programming pulse, a read pulse was applied across the cell in order to measure the read cell current, $I_{\text {cell }}$, and, hence, the resistance, $R_{\text {cell }}$, of the programmed state.

For the considered cell geometry and materials, the maximum temperature during the programming pulse is achieved inside the PC layer, as shown in Fig. 3, rather than at the chalcogenide-heater interface [2]. This is primarily due to the fact that our plug is made of tungsten, which as very low electrical and thermal resistivity when compared to the same parameters of chalcogenide materials. Thus, our device architecture is "self-heating" because gives a much higher contribution to the temperature increase inside the PC volume as compared to the "heater heating" device architecture, where the heater electrical/thermal resistances are comparable to those of the chalcogenide layer. Due to self-heating, we obtain a parallel phase configuration, where a central amorphous 


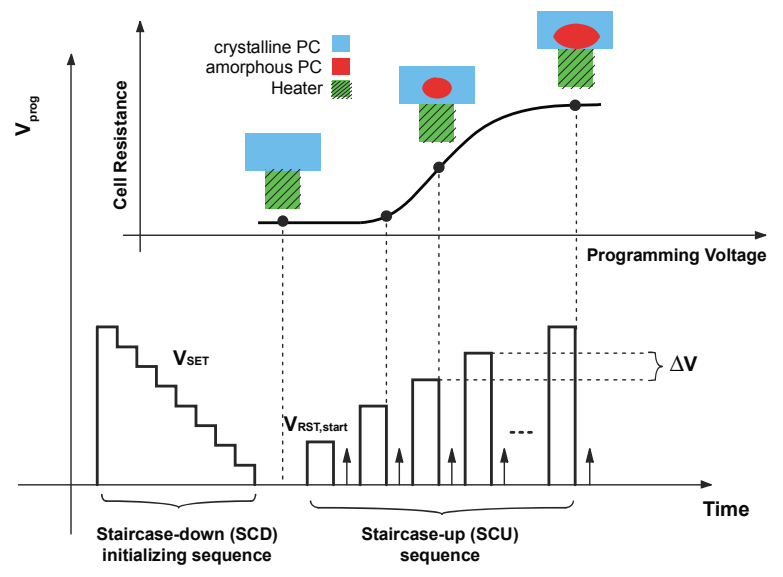

Figure 2. Staircase-up partial-RESET programming algorithm for multilevel storage: sequence of programming pulses (bottom) and qualitative behavior of the PCM cell resistance and phase distribution during partial-RESET (top).

volume is surrounded by crystalline chalcogenide (see Fig. 3). Note that, on the contrary, in typical "heater-heating" devices, we easily obtain a series configuration of the amorphous and crystalline phases [8]. This has important consequences on the ML strategy to be applied, as will be described in the next Section.

\section{MATERIAl COMPARISON \& MULTILEVEL FUNCTIONALITY}

Typical experimental programming curves of different PCM cells integrating GST, GeTe and GeTeN10\% are plotted in Figs. 4 and 5.

More specifically, Fig. 4 and Fig. 5 show the low-field device resistance, $R_{\text {cell }}$, and the read current, $I_{\text {cell }}$, respectively, as a function of the current flowing through the device during programming $\left(I_{\text {prog }}\right)$. For each device, the initial programming voltage, $V_{R S T \text {,start }}$, of the sequence (Fig. 2) was chosen according to the minimum value required to melt the chalcogenide alloy. It can be noticed from Fig. 4, that the SET-to-RESET transition is much sharper in GeTe cells as compared to the case of GeTeN10\% and GST cells. This behaviour is even more evident in Fig. 5, where $I_{\text {cell }}$ decreases from the maximum to the minimum value in an $I_{\text {prog }}$ range of about $5 \mathrm{~mA}$ and $10 \mathrm{~mA}$ for $\mathrm{GeTe}$ and $\mathrm{GeTeN} 10 \%$ cells, respectively, whereas the transition takes place in a larger range $(\sim 30 \mathrm{~mA})$ for GST cells. The above different characteristics may be ascribed to the different electrical and thermal behaviour of the considered PC alloys, as discussed below.

From the thermal viewpoint, the two key parameters are the thermal conductivity and the melting point of the PC alloys. GeTe and doped-GeTe have essentially the same thermal properties [5]. The lower melting temperature of GST $\left(T_{M}=615^{\circ} \mathrm{C}\right)$ with respect to that of $\mathrm{GeTe}$ and $\mathrm{GeTeN} 10 \%$ $\left(T_{M}=720^{\circ} \mathrm{C}\right.$ by differential thermal analysis measurements) compensates for the slightly lower thermal resistance of GSTbased cells and allows obtaining a similar value of melting current for the three alloys considered in this study (the melting current being the $I_{\text {prog }}$ at which $R_{\text {cell }}$ starts to increase). When

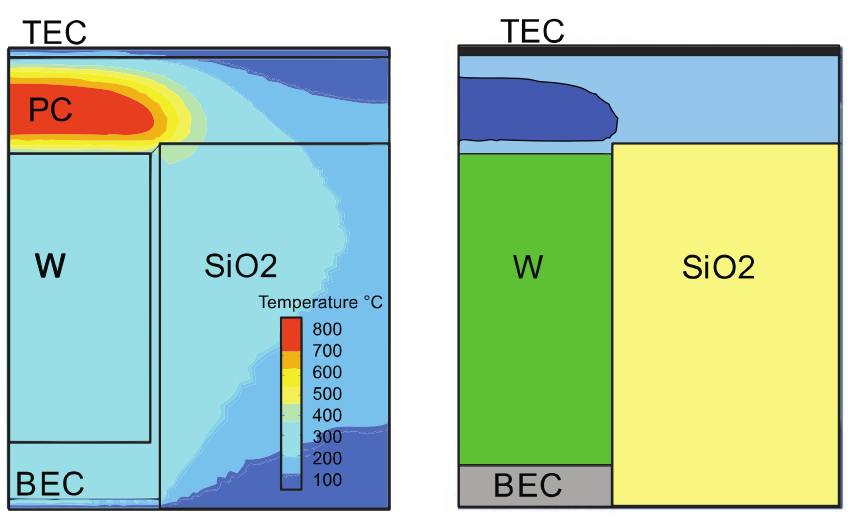

Figure 3. Simulated temperature map inside the considered PCM cells (left). It can be noticed that the maximum temperature is attained inside the chalcogenide layer, which gives rise to a parallel phase configuration (right) in partial-RESET programming.

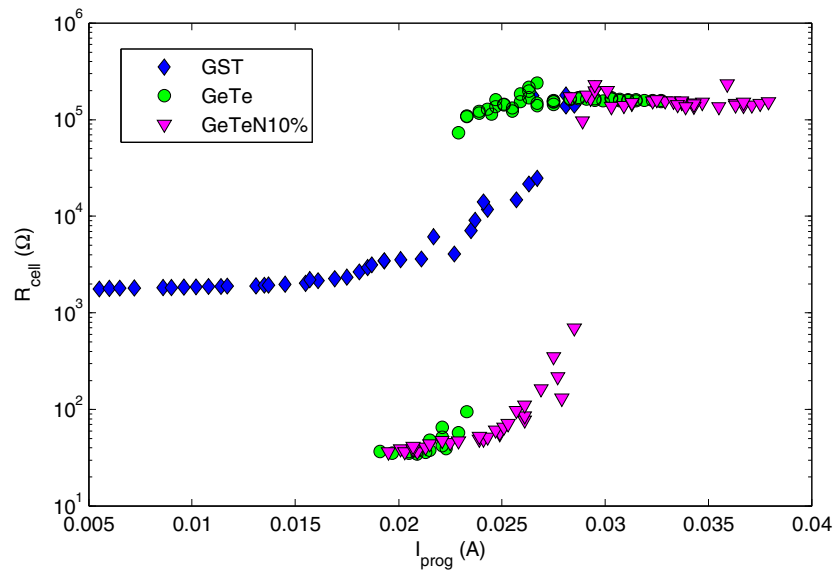

Figure 4. $R_{\text {cell }}-I_{\text {prog }}$ programming curves of PCM cells based on GeTe, GeTeN10\%, and GST.

focusing on the electrical behaviour, three aspects must be considered: crystalline-phase resistivity, amorphous-phase resistivity, and field dependence of the PC layer resistivity.

The resistivities of the two phases affect the shape of the SET-to-RESET curve by determining the width of the programmable current window (i.e., the range from the minimum to the maximum read cell current), and are also partially responsible for the slope of the programming curve. Concerning the programmable window, although the maximum resistance $\left(R_{\text {RESET }}\right)$ is on the order of few hundreds $\mathrm{k} \Omega$ for all the considered materials, the minimum resistance $\left(R_{\mathrm{SET}}\right)$ varies with alloy composition. The values of the SET and the RESET resistance for the considered PC materials as summarized in TABLE I. It can be noticed that the programming window of GST-based cells is narrower with respect to that of the other alloys under study by at least one order of magnitude. In this respect, it is worth to point out that, in the case of ML storage, a narrower programmable window 


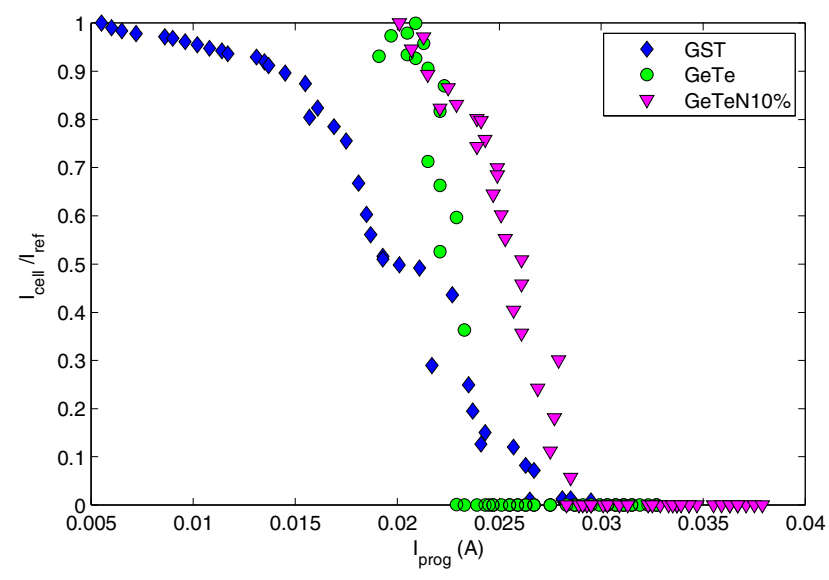

Figure 5. $I_{\text {cell }} I_{\text {prog }}$ programming curves of PCM cells based on GeTe, GeTeN10\%, and GST (read cell currents are normalized with respect to the cell currents in the SET state).

TABLE I. Set-State and Reset-State Device Resistance for DIFFERENT CHALCOGENIDE ALLOYS

\begin{tabular}{|c|c|c|c|}
\hline & \multicolumn{3}{|c|}{ Chalcogenide Composition } \\
\cline { 2 - 4 } & GeTe & GeTeN10\% & GST \\
\hline$R_{\text {SET }}[\Omega]$ & 40 & 50 & $2 \mathrm{k}$ \\
\hline$R_{\text {RESET }}[\Omega]$ & $\sim 200 \mathrm{k}$ & $\sim 200 \mathrm{k}$ & $\sim 200 \mathrm{k}$ \\
\hline
\end{tabular}

requires higher accuracy both in reading operation, due to the reduced spacing available between adjacent programmed states, and in programming operation, due to the need for allocating the adequately spaced programmed states in a narrower range [6].

The field dependence of the PC layer resistivity has a significant impact on the programming operation of our cells, since they are essentially programmed thanks to self-heating. Indeed, heater heating gives a very small contribution to the PC layer temperature due to the low resistance and the low thermal efficiency of the heater. In self-heating PCM cells, the temperature reached inside the PC layer mainly depends on the power developed inside the PC layer, differently from the case of heater-heating cells, where the heating power is essentially controlled by the heater. Several chalcogenide alloys show a non-ohmic behavior characterized by an increase of the electrical conductivity with increasing electric field. From the experimental current-voltage characteristics in the programming region of our PCM cells shown in Fig. 6, it is possible to notice that GeTe has an ohmic behavior, whereas GST and $\mathrm{GeTeN} 10 \%$ are non-ohmic materials.

The intercept of the extrapolated lines with the $V$-axis define the parameter $V_{H}$, which is related to the non-ohmic part of the chalcogenide resistance: $V_{H}$ is highest $(\sim 1 \mathrm{~V})$ for GST cells, whereas it is about $0.4 \mathrm{~V}$ for $\mathrm{GeTeN} 10 \%$ cells. The slope of the extrapolated lines in Fig. 6 corresponds to the inverse of the ohmic part of the PCM cell resistance which accounts for the ohmic behavior of the chalcogenide layer above the hold voltage (if any), $R_{\Omega}$, plus the resistance of the heater, $R_{H}$ and the metal lines, $R_{M}$.

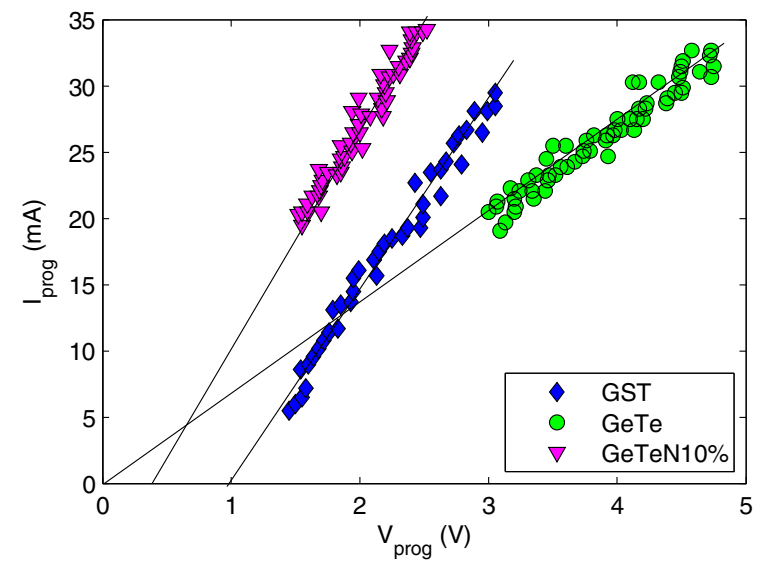

Figure 6. $I_{\text {prog }}-V_{\text {prog }}$ curves of PCM cells with GeTe, GeTeN10\%, and GST.

Being the heater contribution to the temperature increase inside the PC material negligible in our devices, the maximum temperature, $T_{\max }$, obtained inside our self-heating cells during programming can be written as:

$$
T_{\max }=R_{t h, P C} P_{P C}
$$

where $R_{t h, P C}$ is the equivalent thermal resistance the PC layer and $P_{P C}$ is the electrical power developed inside the PC material. Then, when the chalcogenide shows a purely-ohmic behavior inside the programming region, the temperature $T_{\max }$ increases quadratically with the current flowing through the cell, since $P_{P C}$ can be written as $P_{P C}=R_{\Omega} I_{\text {prog }}{ }^{2}$. On the other hand, in purely $V_{H}$-alloys, $T_{\max }$ increases linearly with $I_{\text {cell }}$, since we have $P_{P C}=V_{H} I_{\text {prog }}$ [8]. When considering a generic $\mathrm{PC}$ material, $P_{P C}$ is the sum of both contributions:

$$
P_{P C}=V_{H} I_{\text {prog }}+R_{\Omega} I_{\text {prog }}^{2}
$$

The developed electrical power in the cases of purely-ohmic and purely $V_{H}$-alloy is schematically depicted in Fig. 7. It can be observed that, in the region of high current values (like in the partial-RESET current range), the range from $P_{\text {melt }}$ (the melting power) to $P_{\mathrm{RST}}$ (the power required to obtain the maximum resistance) corresponds to a programming current range that is wider for the $V_{H}$-alloy due to the linear dependence of the power upon current. This results in a larger transition region of the SET-to-RESET programming curve (exactly what happens in $\mathrm{GeTeN} 10 \%$ w.r.t. GeTe), which simplifies ML storage since less accuracy is required for the programming pulse amplitude. The above different behaviors are apparent in Fig. 8, where the power developed inside the PC layer is plotted and compared to the above model for cells based on GeTe (ohmic material) and GeTeN10\% ( $V_{H}$-alloy with a very small $R_{\Omega}$ ). As expected, $P_{P C}$ increases faster with the programming current in the case of GeTe cells, which leads to a narrower transition region of the programming curve (Fig. 4). This hinders the capability of programming intermediate levels with the required accuracy, making our GeTe-cells unsuitable for ML programming. 


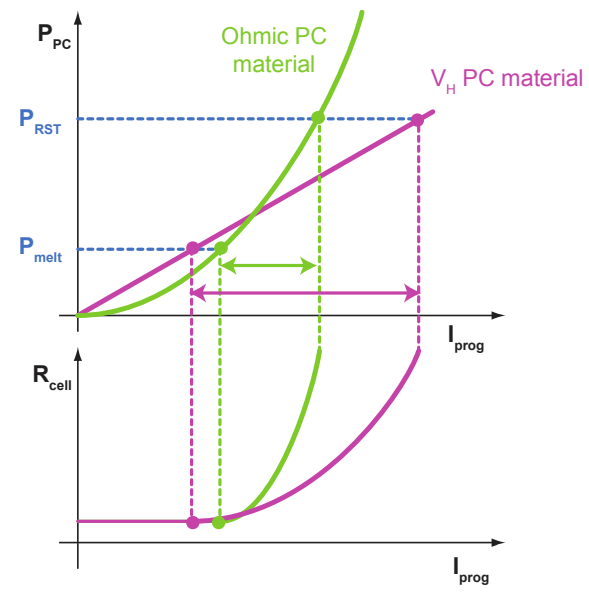

Figure 7. Schematic representation of the self-heating behavior of cells based on ohmic chalcogenide and $V_{H}$-alloys: programming power (top) and corresponding $R_{\text {cell }} I_{\text {prog }}$ programming curve (bottom).

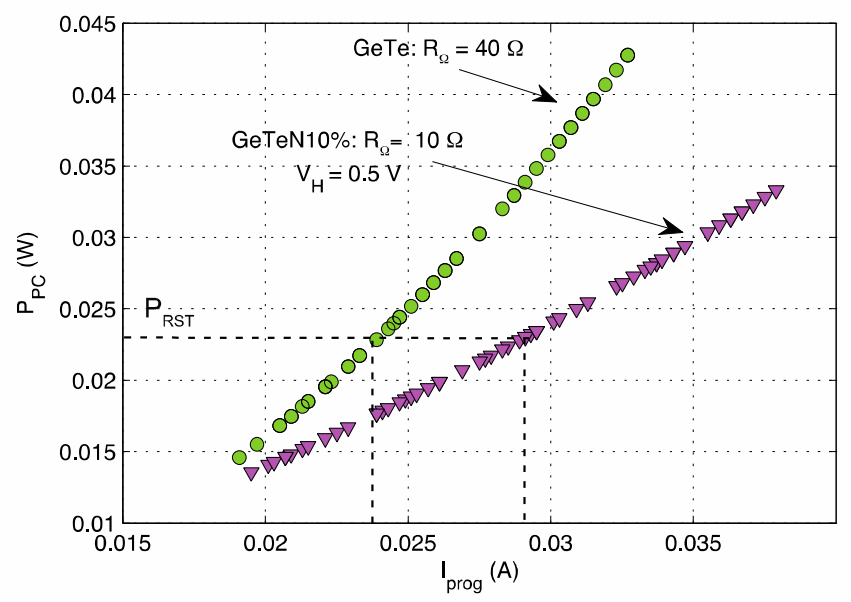

Figure 8. Programming power, $P_{P C}$, developed inside the $P C$ layer calculated with Eq. (2). $R_{\Omega}$ and $V_{H}$ of the two alloys are estimated from their respective $V_{\text {prog }}-I_{\text {prog }}$ curves.

TABLE II. Resistance SPacing of the PRogrammed Levels for GST AND GeTeN10\%

LeVel 00 LeVel 01 LeVel 10 LeVel 11

\begin{tabular}{|c|c|c|c|c|}
\hline GST & $2 \mathrm{k} \Omega$ & $3 \mathrm{k} \Omega$ & $7 \mathrm{k} \Omega$ & $100 \mathrm{k} \Omega$ \\
\hline GeTeN10\% & $65 \Omega$ & $90 \Omega$ & $180 \Omega$ & $100 \mathrm{k} \Omega$ \\
\hline
\end{tabular}

We experimentally demonstrated the feasibility of ML storage in $\mathrm{GeTeN} 10 \%$ cells by programming four linearly-spaced current levels (Fig. 9). The corresponding values of cell resistance for the programmed levels are summarized in TABLE II. It is worth noticing that, first, the width of the programmable current window is much higher in the case of $\mathrm{GeTeN10 \%}$ due to the lower resistivity of crystalline GeTe with respect to GST [5], thus leading to a higher spacing between adjacent levels and higher read current, for a chosen value of read voltage across the cell. Second, being the

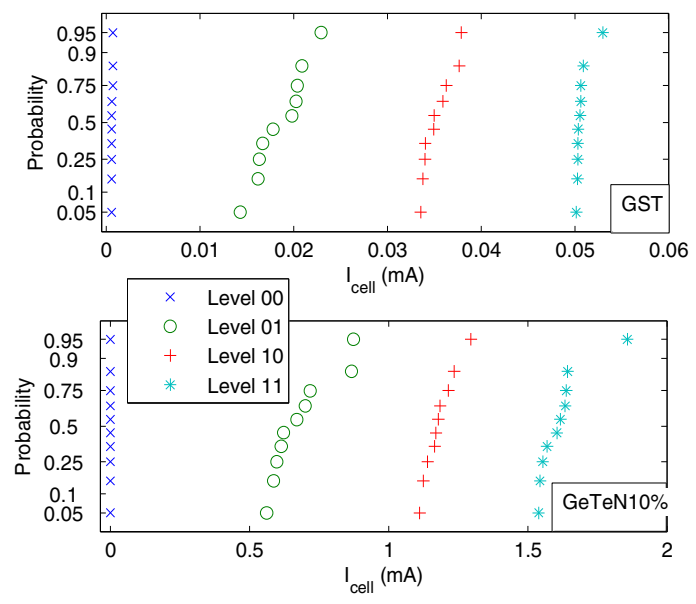

Figure 9. Probability distributions of four linearly-spaced resistance levels for GST (top) and GeTeN10\% (bottom). This distribution are obtained from a small population of 10 cells. The cell read voltage was set to $100 \mathrm{mV}$.

resistance levels very near to the SET state, they should suffer less of the drift parasitic effect [6], with ensuing benefit for the stability in time of the states of information.

\section{CONCLUSIONS}

In this work, the impact of chalcogenide alloy electrical properties on the feasibility of ML storage in self-heating PCM cells has been assessed. GST and GeTeN10\% present a wider transition region of the SET-to-RESET programming curve with respect to GeTe, which facilitates ML programming. This is ascribed to the different electrical behavior of the PC alloys (GeTe is an ohmic alloy, whereas GeTeN10\% and GST are $V_{H^{-}}$ alloys). In addition, GeTeN10\% has a wider programmable window than GST. Then, among the considered alloys, GeTeN10\%, first, and GST, second, give the best performance from the viewpoint of ML programming.

\section{ACKNOWLEDGMENT}

This work has been partially supported by the Italian MIUR in the frame of its National FIRB Project RBAP06L4S5.

\section{REFERENCES}

[1] R. Bez, et al., "Phase change memory development trends," Proc. of Int. Memory Workshop, pp. 1-4, May 2010.

[2] G. W. Burr, et al., "Phase change memory technology," J. of Vacuum Science \& Technology B: Microelectronics and Nanometer Structures, vol. 28, no. 2, pp. 223-262, March 2010.

[3] W. Czubatyj et al., "Nanocomposite Phase-Change Memory alloys for very high temperature data retention," Electron Dev. Lett., Aug 2010, vol 31, n. 8,pp. 869-871.

[4] G. Betti Beneventi, et al., "On carbon doping to improve GeTe-based phase-change memory data retention at high temperature," Proc. of Int. Memory Workshop, pp. 1-4, May 2010.

[5] A. Fantini, et al., "N-doped GeTe as performance booster for embedded phase-change memories," Proc. of Int. Electron Device Meeting, Dec. 2010.

[6] S. Braga, et al.. "Voltage-driven partial-RESET multilevel programming in Phase Change Memories,". IEEE Trans. on Electron Devices, vol. 57, no. 10 , pp. 1-8, Oct. 2010.

[7] D.-H. Kang et al., " Two-bit Cell Operation in Diode-Switch Phase Change Memory Cells with 90nm Technology", VLSI Symp. Tech Dig., pp. 98-99, 2008.

[8] A. Itri et al., "Analysis of phase-transformation dynamics and estimation of amorphous-chalcogenide fraction in phase-change memories", Proc. of Int. Reliability Physics Symp., pp.209-215, 2004. 